\title{
Professional Socialization and Attitudes Towards Interprofessional Collaboration Among Graduate Social Work and Health Professions Students
}

\author{
Allison West \\ Shari Miller \\ Judith Leitch
}

\begin{abstract}
Although there is an aggressive push towards interprofessional collaboration in higher education as well as in practice, the traditional culture and organization of higher education, as well as the need for and history of disciplinary distinction, may impede these efforts. Using an online survey, this study explored the relationship between professional socialization of 157 graduate students in four disciplines and their perceptions and attitudes about interprofessional collaboration. Results indicate that first year students had more positive perceptions and attitudes about interprofessional collaboration than more advanced students. Furthermore, social work students perceived themselves as having lower prestige than graduate-level nursing, pharmacy, and medical students. These findings suggest that, unless managed strategically, professional socialization may diminish positive perceptions and attitudes towards interprofessional collaboration. Thus, social work educators should pay careful attention to the role of professional socialization and how it is manifest in both the explicit and implicit curriculum.
\end{abstract}

Keywords: Interprofessional education; interprofessional collaboration; professional socialization

Collaborative interprofessional practice (IPP) is increasingly viewed as a critical means of addressing complex multidimensional problems in health and human services. In 2010 the World Health Organization (WHO) published a Framework for Action on Interprofessional Education \& Collaborative Practice specifically recognizing collaboration between professions as "an innovative strategy that will play an important role in mitigating the global health crisis" (p.7). The Institute of Medicine (IOM; 2003), the American Public Health Association (2009), and the Council on Social Work Education (2012) have also articulated strong support for IPP. As an emphasis on IPP has gained ground, a necessary focus on education for IPP has emerged. Centers, institutes, and other centralized structures focused on effective education for IPP have been proliferating at universities across the U.S. In 2012, the importance of this endeavor was underlined by the Health Resources Services Administration (HRSA) of the U.S. Department of Health and Human Services. HRSA, in conjunction with four private foundations, awarded $\$ 12$ million to the University of Minnesota's Academic Health Center to "provide national leadership in the field of interprofessional education and collaborative practice among health professionals" via the establishment of the National Coordinating Center for Interprofessional Education and Collaborative Practice - or CC-IPECP (HRSA, 2012, para.1).

Allison L. West, PhD, MSW is Assistant Scientist at the Johns Hopkins Bloomberg School of Public Health, Baltimore, MD 21205. Shari Miller, PhD, MSW is Associate Professor, School of Social Work, University of Georgia, Athens, GA

30602. Judith Leitch, MSW is a PhD student at the School of Social Work, University of Maryland, Baltimore, MD 20201. 
Evidence suggests that IPP is associated with lower costs, decreased lengths of hospital stay, reduced medical errors, and enhanced quality of patient care (IOM, 2003). Research has consistently demonstrated that interprofessional collaboration is also essential in translating findings from research to practice and improving client outcomes (Curran, Sharpe, Forestall, \& Flynn, 2008; Mabry, Olster, Morgan, \& Abrams, 2008; Syme, 2008). In research settings, interprofessional collaboration creates opportunities for improved access to funds, expertise, and instrumentation; it has also been shown to enhance researchers' productivity and the extent to which they enjoy their work (Van Rijnsoever \& Hessels, 2011).

In the context of the global arena, social workers are well-suited to be leaders in teambased collaborative processes. Yet, there is little systematic research regarding social worker's participation in and contributions to interprofessional endeavors. Few studies have examined social workers' attitudes towards or involvement in interprofessional collaboration (Curran et al., 2008; Ko, Bailey-Kloch, \& Kim, 2014; Leipzig et al., 2002) or the idea of differential professional cultures and ways in which they have bearing on interprofessional relationships (Hall, 2005).

\section{Interprofessional Practice and Social Work}

There is a growing body of literature that supports the value of interprofessional collaboration within the field of social work (Bronstein, Mizrahi, Korazin-Korosy, \& McPhee, 2010; Matto \& Strolin-Goltzman, 2010; Sharland, 2012). The practice of integrating research from other disciplines is increasingly encouraged in light of mounting evidence linking the biological and social sciences. Matto and Strolin-Goltzman (2010), for example, suggested that increased integration of neuroscience into social work will help advance practice-based research. In addition, studies have demonstrated that inclusion of social work services increases effectiveness in health care prevention and treatment (Freshman, Rubino, \& Chassiakos, 2010; Kenny, Sparks, \& Jackson, 2007).

Some research has examined factors associated with interprofessional collaboration among social work and other allied health professional students. Results from two studies demonstrated that social work students had more positive attitudes towards teamwork than medical students (Curran et al., 2008; Leipzig et al., 2002). Hawk et al. (2002) found that attitudes towards interprofessional collaboration differed among graduate professional students depending on their program of study but not race, age, or gender. However, in a study of research collaboration, Van Rijnsoever and Hessels (2011) found that female students were more likely than male students to engage in collaboration. The vast majority of social workers are female (U.S. Bureau of Labor Statistics, 2014), thus gender may be a confounding factor in studies examining collaboration. Further, when considering the cultural and historical contexts within which professions emerge and evolve, the very reasons why there are such notable gender disparities among the population of social workers in and of itself may have bearing on attitudes toward collaboration. 


\section{Interprofessional Education and Social Work}

Growing appreciation for the value of interprofessional collaboration in social work practice has led to a subsequent increase in interest in interprofessional education (IPE). With the publication of the Council on Social Work Education's (CSWE) 2015 Educational Policy Accreditation Standards (EPAS), interprofessional collaboration was codified as a necessary element of social work education and practice. IPE is a pedagogical approach that involves educators and learners from two or more professional disciplines learning about, from, and with each other to develop attitudes, knowledge, and skills with the ultimate goal of improving collaboration and client care (Barr, Freeth, Hammick, Koppel, \& Reeves, 2006; Buring et al., 2009; WHO, 2010). The authors of the WHO Framework (2010) suggest that IPE can play a significant role in the development of a collaborative practice-ready workforce, which can help reduce barriers to optimal care faced by health systems around the world. IPE does not simply entail students from multiple disciplines receiving the same learning experience, nor is it faculty from one field teaching or mentoring students from another; rather, it is a fully integrated or shared learning and decision-making process (Buring et al., 2009). Whereas interest in promoting IPE within BSW and MSW programs appears to be increasing, evidence suggests that few social work programs incorporate interprofessional learning as an integrated component of the curriculum (Bronstein et al., 2010; McPherson, Headrick, \& Moss, 2001; Reeves et al., 2010).

A literature search using the terms "social work" and "interprofessional education" identified numerous articles concerning specific curricula or approaches to IPE. Though most of these articles are exclusively conceptual or descriptive, they represent efforts at organized collaboration between social work and a wide variety of disciplines including nursing, medicine, dentistry, occupational therapy, physical therapy, pharmacy, chaplaincy, education, nutrition, and law. In social work, dual degree programs and/or formal teaching arrangements are particularly common approaches to IPE (Bronstein et al., 2010; Simms, 2011). Other popular strategies found in the social work literature include one-time seminars and year-long field education experiences, although examples of more longitudinal, integrated programs also exist (Pecukonis et al., 2013). Yet despite growing interest in IPE, there remains a dearth of evidence regarding the most effective format and structure for IPE learning experiences. A recent systematic review of studies examining the effectiveness of IPE programs demonstrated mixed results; whereas some programs evidenced positive outcomes, others appeared to have no impact on health care processes or patient outcomes (Reeves et al., 2010).

\section{Professional Socialization and Social Work}

When considering IPE, it is also essential to recognize that professions are cultures unto themselves, replete with values, attitudes, and norms into which practitioners are socialized (Miller, 2010, 2013). Given the complex matrix of factors that define each profession's culture, and the likely differences between professional cultures, interprofessional efforts may then become exponentially more complex. In order to better understand these interprofessional efforts, and how to deploy educational and practice 
models effectively in the context of current and potentially shifting structures, the need to factor in the dynamics of professional socialization becomes essential.

Professions are characterized as cultures into which students and practitioners are continually socialized over the course of their careers (Barretti, 2004b; Miller, 2010, 2013). In the context of IPE and practice, consideration for the intersection of these professional cultures, and the need to understand them with proficiency, if not fluency, becomes elemental. The process of becoming professionally socialized emerges through a complex system of formal and informal processes and relationships (Barretti, 2004a) that begin before formal professional education and continue as professionals make dynamic adaptations in practice (Barretti, 2004b; Miller, 2010, 2013; Shuval, 1980). Notions of professional socialization have been infused in the culture of social work education since its inception; however, it was not until 2008 that the construct was articulated in CSWE's educational policy and accreditation standards (Miller, 2013). Professional socialization is generally considered a positive and vital process. Studies of doctoral students have indicated that professional socialization is essential to a successful graduate school experience and that inadequate socialization may lead to attrition (Clark \& Corcoran, 1986).

\section{A Framework for Understanding Professional Socialization}

In 2010, Miller presented a multi-dimensional framework for understanding the professional socialization of social workers adapted from a long-developing and wellestablished body of knowledge in medicine and nursing. This adapted theoretical model incorporates aspects of both symbolic interactionism and structural functionalism, suggesting that socialization results from both intended and unintended influences, and that role modeling and critical self-reflection may play key roles. The framework organizes professional socialization as a process of ongoing change that includes three stages: presocialization, formal socialization, and practice after formal socialization. Pre-socialization is inclusive of the influence of both prior (primary, early) socialization and anticipatory socialization, both of which have bearing on the choice to pursue formal social work education. As students anticipate entering the culture of social work, they begin a process of internal and external reorganization around role identity, attitudes, norms, and values that they believe are emblematic of the professional culture into which they are about to enter. According to the literature, this phase seems to have a strong relationship to the continued process of professional socialization (Abbott, 1988; Bargal, 1981; Barretti, 2004b; Miller 2010, 2013; Shuval, 1980).

Formal socialization encompasses the period of time during which a student is engaged in earning a social work degree. This phase is influenced by a number of factors including course content and relationships with others, including professors, field instructors, colleagues, clients, and fellow students. The third phase, practice after formal socialization, recognizes that professional socialization is not a concrete and static outcome, but is a process with discrete cultural components that continues to unfold over time. This phase presumes that practitioners have adequate agency and are active decisionmakers in how they locate themselves in the culture. This phase also presumes that practitioners are nimble in situationally adapting to varying practice contexts. Research 
suggests there are number of potential factors that relate to how social workers are professionally socialized and ways in which that socialization influences chosen practice pathways and ways of engaging in practice (Miller, 2013).

With this framework as a backdrop, there are additional factors to consider, in particular when trying to understand the complex interactions necessary for effective interprofessional collaboration. Interactions with people outside the field of social work may also impact the socialization process. For example, interactions with professionals from other disciplines may influence how a social work student views her/himself as well as others within her/his profession. Miller's (2010) framework suggests that, through the lens of symbolic interaction, students (and later practitioners) construct their professional identity in some ways by choosing who to include in their generalized other. This professional generalized other could include colleagues, peers, classroom instructors, field instructors, and clients from within the culture of social work, and it can also include other professionals with whom they have contact in the field. The framework suggests that social workers actively make choices about what messages and information to reject or incorporate, and those choices can also evolve over time.

Building in an explicit facet of role theory may help inform our understanding of role expectations as well as the value each profession places on autonomy within an interprofessional setting. Although a strong sense of professional role is an imperative component of both professional socialization and successful collaboration, placing too high a value on one voice may inhibit effective collaboration as well as effective client care (Clark, 1997). Because social workers often work in interprofessional teams in which theirs may not be the dominant voice, social work educators are challenged to nurture a strong sense of autonomy, respect for the contributions of other disciplines, and a well-honed ability to effectively negotiate some of these challenges.

\section{The Nexus of Professional Socialization and Interprofessional Collaboration}

If not carefully facilitated, instead of encouraging understanding and interactions with other disciplines, the process of professional socialization could widen the gap between social workers and other types of professionals and thus may contribute to the creation of silos of learning, practice, and research (Syme, 2008). Discipline-specific boundaries are often further solidified via involvement in professional societies and professional publications (Boden, Borrego, \& Newswander, 2011). Thus, a natural tension appears to exist between discipline-specific socialization and the need for interprofessional collaboration.

Notably, two social work scholars have written about the tension that lies at the intersection of disciplinarity and interdisciplinarity. Sharland (2012) suggested that the distinction between disciplinarity and interdisciplinarity is muddled by the heterogeneous nature of the social work profession, a general lack of internal cohesion as a profession, as well as confusion as to the nature of social work as a discipline. A portion of this confusion stems from the heavy reliance on knowledge from other disciplines, as well as social workers' presence in a diverse range of practice settings. Couturier, Gagnon, Carrier, and Etheridge (2008) took a more philosophical stance, asserting that epistemological 
differences between disciplines are an "unavoidable condition" of collaboration, and that the true value of interdisciplinarity stems from a meeting of epistemologies that results in a transformation in "the very act of knowing" (p. 341). When considering the evolution of professional cultures, the role of epistemological orientation has direct relevance to professional socialization. Thus disciplinarity and interdisciplinarity can be understood as two sides of the same coin, with one unable to exist without the other.

A better understanding of how attitudes towards interprofessional collaboration change over the course of a graduate program will provide valuable insight into current educational practices as well as potential curricular needs. Such insight is crucial if we hope to equip social workers with the requisite skills and abilities to effect significant positive change within multidisciplinary settings. The purpose of this exploratory study was to examine the relationship between stages of professional socialization of social workers and attitudes towards interprofessional collaboration. This study explored differences in attitudes towards collaboration between the first (pre-socialization) and later years of graduate study (formal socialization) of different professional graduate-level disciplines. The study addressed the question: Do stage of socialization and graduate program of study predict students' attitudes towards interprofessional collaboration? Based on the theoretical framework of this study, we expected that as graduate students became increasingly socialized to their disciplines over time, greater professional socialization would be associated with less positive attitudes towards interprofessional collaboration.

\section{Method}

\section{Participants and Procedures}

This exploratory study was part of a larger study that examined attitudes, perceptions, and experiences related to interprofessional collaboration among graduate professional students. An Internet questionnaire was distributed through a student government association to all students enrolled in graduate professional degree programs at a large public Mid-Atlantic university. Because the focus of this research was on collaboration among professionals from allied health care disciplines, the sampling frame was limited to a subset of students who were pursuing masters or professional doctorate degrees in nursing, social work, pharmacy, or medicine $(n=3,396)$. The survey was disseminated via an e-mail link to the entire student population during the first week of the fall semester with a reminder and link distributed two weeks later. No incentive was provided to students for survey completion. Of the students who met the eligibility criteria, 228 completed the survey (response rate of 6.7\%). Seventy-one cases were removed from the analyses because students failed to clearly indicate the year in which they entered the program, whether they were full or part-time students, and/or they did not complete at least $50 \%$ of the items on the Interdisciplinary Education Perception Scale (IEPS; Luecht, Madsen, Taugher, \& Petterson, 1990). The final sample included 157 graduate professional students. Although the response rate was low, the sample size was deemed sufficient in light of the exploratory nature of this research. This is one of the first studies to begin to consider the relevance of professional socialization to IPE and provides a useful foundation for future research. 


\section{Measures}

Demographics. Respondent's self-reported age was a continuous variable included as a covariate based on prior research and theory. Programs of study (social work, medicine, nursing, pharmacy) were dummy-coded, with social work serving as the reference group.

Professional socialization. Though this construct overall is quite complex, as a beginning point for the purposes of this exploratory research, year of study served as a proxy for professional socialization, given that there is an expectation that socialization to a profession will develop over time. Respondents indicated the year that they began their graduate program (2009-2012) and whether they were attending as full- or part-time students. Part-time students in the first or second year of study were re-coded as being in Year One of their graduate program, as this label was felt to represent the level and number of courses in which they would be enrolled, time spent in coursework, and interaction with student peers. Similarly, part-time students who entered in years 2009 or 2010 were recoded as Year Two.

Perceptions of interprofessional collaboration. The Interprofessional Education Perception Scale (IEPS; Luecht et al., 1990) is a commonly used self-report perceptual/attitudinal inventory designed to assess concepts related to successful interprofessional teamwork. The original scale was comprised of 18 items divided into four subscales: Competency and Autonomy, Perceived Need for Cooperation, Perception of Actual Cooperation, and Understanding Others' Value. Subsequent research seeking to refine the measure has produced alternate versions which have demonstrated stronger psychometric properties (Leitch, 2014; McFadyen, Maclaren, \& Webster, 2007; Vaughan, Macfarlane, Dentry, \& Mendoza, 2014; Williams \& Webb, 2013).

Recently, Leitch (2014) proposed a 16-item version of the IEPS that is grounded in current theory and research in the area of interprofessional collaboration. This revised version contains three subscales: Competency and Autonomy, Cooperation, and Prestige. The Competency and Autonomy subscale contains items designed to tap feelings about competence of self and others within their profession such as, "Individuals in my profession trust each other's professional judgment." The Cooperation subscale contains items tapping attitudes about working with other professions such as, "Individuals in my profession need to cooperate with other professions." Notably, this is the first iteration of the IEPS to include a scale that isolates perceived prestige as a factor presumed to influence collaboration. An example item from the Prestige subscale is, "Individuals in other professions respect the work done by my profession." The instrument is a Likert-type scale with response options ranging from "strongly disagree" (1) to "strongly agree" (6). Items within each subscale are added to create a sum score, with higher scores indicating more positive perceptions of interprofessional collaboration. Subscale scores were used as dependent variables in analyses in lieu of the total scale score to allow for a more nuanced understanding of attitudes towards collaboration. In this study, the IEPS subscales demonstrated good internal consistent reliability for Competency and Autonomy $(\alpha=.82)$ and Prestige $(\alpha=.80)$, with lower reliability for Cooperation $(\alpha=.64)$. 


\section{Data Analysis}

A series of three two-step hierarchical multiple regression analyses assessed the effects of age, program of study, and year of study on each of the three subscales of the IEPS: Competency and Autonomy, Prestige, and Cooperation. Age and program of study were entered in step one. Year of study was entered in step two. For cases with missing data on individual IEPS items, item scores were imputed using regression imputation. Statistical assumptions for multiple regression analyses were tested and met for all variables. Data analyses were conducted using SPSS v. 20.

\section{Results}

The mean age for all 157 students was 30.6 years ( $S D=10.05$, Min=19, Max=59). Just under half of the students in the sample were in their first year of graduate study $(n=74$, $47.1 \%)$, and just over half were in their second, third, or fourth year of study ( $n=83,52.9 \%)$ (see Table 1). Medical students had the highest Competency and Prestige scores while Pharmacy students had the highest scores for Cooperation (see Table 2). Bivariate correlations for each of the IEPS subscales, age, program of study, and year of study are provided in Table 3.

Table 1. Participant Characteristics by Program of Study $(n=157)$

\begin{tabular}{lcccc}
\hline & $\begin{array}{c}\text { Social Work } \\
(\boldsymbol{n}=\mathbf{5 2})\end{array}$ & $\begin{array}{c}\text { Medicine } \\
(\boldsymbol{n}=\mathbf{2 4})\end{array}$ & $\begin{array}{c}\text { Nursing } \\
(\boldsymbol{n}=\mathbf{3 6})\end{array}$ & $\begin{array}{c}\text { Pharmacy } \\
(\mathbf{n = 4 5})\end{array}$ \\
\hline & $f(\%)$ & $f(\%)$ & $f(\%)$ & $f(\%)$ \\
\hline Gender (Male) & $2(3.9)$ & $4(16.7)$ & $2(5.6)$ & $8(18.2)$ \\
$\begin{array}{l}\text { Enrollment Status (Full-Time) } \\
\text { Year* }\end{array}$ & $46(88.5)$ & $24(100)$ & $17(47.2)$ & $37(84.1)$ \\
$\quad$ & & & \\
$\quad$ First & $26(50)$ & $8(33.3)$ & $20(55.6)$ & $20(44.4)$ \\
$\quad$ Second, Third, or Fourth & $26(50)$ & $16(66.6)$ & $16(44.4)$ & $25(56.8)$ \\
\hline
\end{tabular}

*Year incorporates FT/PT status

Table 2. IEPS Subscale Scores by Program of Study $(n=157)$

\begin{tabular}{lcccc}
\hline Subscale & $\begin{array}{c}\text { Social Work } \\
\boldsymbol{M}(\boldsymbol{S D})\end{array}$ & $\begin{array}{c}\text { Medicine } \\
\boldsymbol{M}(\boldsymbol{S D})\end{array}$ & $\begin{array}{c}\text { Nursing } \\
\boldsymbol{M}(\boldsymbol{S D})\end{array}$ & $\begin{array}{c}\text { Pharmacy } \\
\boldsymbol{M}(\boldsymbol{S D})\end{array}$ \\
\hline Competency & $28.5(3.9)$ & $30.8(2.8)$ & $29.4(3.9)$ & $30.4(3.7)$ \\
Prestige & $14.4(3.2)$ & $20.0(2.5)$ & $17.6(3.8)$ & $16.7(2.9)$ \\
Cooperation & $29.8(3.3)$ & $28.4(2.4)$ & $30.9(3.1)$ & $31.3(2.4)$ \\
\hline
\end{tabular}


Table 3. Correlation Matrix for Model Variables

\begin{tabular}{|c|c|c|c|c|c|c|c|c|c|}
\hline & 1 & 2 & 3 & 4 & 5 & 6 & 7 & 8 & 9 \\
\hline 1. Age & 1 & & & & & & & & \\
\hline 2. First year of study & .09 & 1 & & & & & & & \\
\hline 3. Nursing & $.49^{* *}$ & .09 & 1 & & & & & & \\
\hline 4. Medicine & $-.20^{*}$ & -.12 & $-.23^{* *}$ & 1 & & & & & \\
\hline 5. Pharmacy & $-.17^{*}$ & -.03 & $-.35^{* *}$ & $-.27^{* *}$ & 1 & & & & \\
\hline 6. Social Work & -.12 & .04 & $-.38^{* *}$ & $-.30^{* *}$ & $-.45^{* *}$ & 1 & & & \\
\hline 7. Competency \& Autonomy & -.02 & .12 & -.03 & .14 & .13 & $-.20^{*}$ & 1 & & \\
\hline 8. Prestige & .13 & $.22^{* *}$ & .14 & $.39^{* *}$ & .01 & $-.43^{* *}$ & $.39^{* *}$ & 1 & \\
\hline 9. Cooperation & .14 & .14 & .12 & $-.27^{* *}$ & $.22^{* *}$ & -.11 & $.56^{* *}$ & $.24^{* *}$ & 1 \\
\hline
\end{tabular}

$* p \leq .05, * * p \leq .01$ (2-tailed).

\section{Competency and Autonomy}

Results from multiple regression analyses are presented in Table 4. In step one, age and program of study accounted for only $5.6 \%$ of the variance in Competency and Autonomy, $F(4,152)=2.25, p=.07$. Adding year of study to the model explained an additional $2.2 \%$ of the variance. Although the change in $R^{2}$ from step one to step two was not significant, the final model including all predictors was significant, $F(5,151)=2.55$, $p=.03$. Results from the final model indicated that medical students, $B=2.29, t=2.49$, $p=.01$, and pharmacy students, $B=1.86, t=2.46, p=.02$, scored higher on this subscale relative to social work students after controlling for age and year of study. There were no differences between nursing and social work students. The positive coefficient for year of study suggested that there was a trend for first year students to score higher than second year students on the competency and autonomy subscale after controlling for age and program of study, $B=-1.13, t=-.15, p=.06$.

Table 4. Unstandardized Multiple Regression Results $(n=157)$

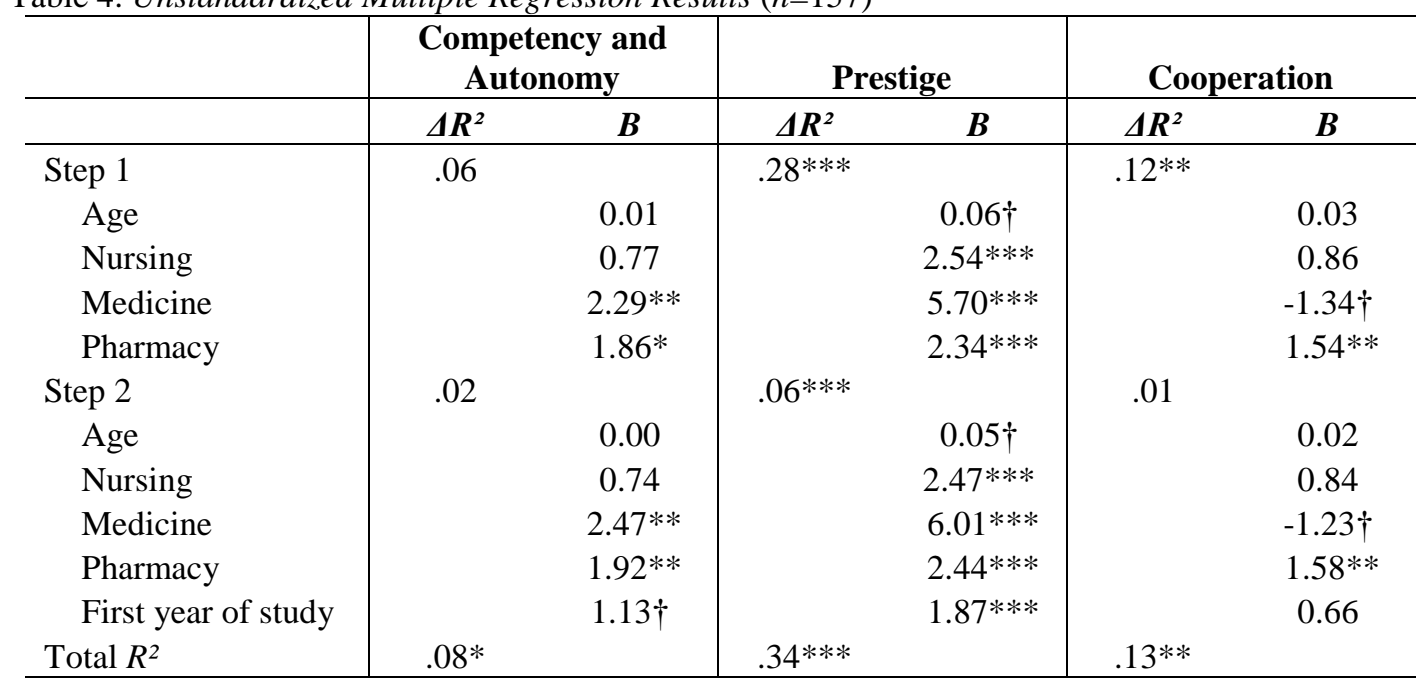

Note. Social work is the reference group.

Note. Coefficients are from final the model including all predictors.

$\dagger p \leq .10, * p<.05, * * p<.01, * * * p \leq .001$ 


\section{Prestige}

For the Prestige subscale, in step one age and program of study accounted for $28 \%$ of the variance, resulting in an overall significant model, $F(4,152)=14.77, p<.001$. Adding year of study explained an additional $6.4 \%$ of variance and resulted in a significant change in $R^{2}$ and a significant model overall, $F(5,151)=15.84, p<.001$. Results from the final model indicated that medical students, $B=6.01, t=7.97, p<.001$, pharmacy students, $B=2.44, t=3.96, p<.001$, and nursing students, $B=2.47, t=3.44, p=.001$, scored higher on the Prestige subscale relative to social work students after controlling for age and year of study. In addition, first year students scored significantly higher on prestige than second year students on this subscale after controlling for age and program of study, $B=1.87$, $t=3.84, p<.001$.

\section{Cooperation}

For the model predicting scores on the Cooperation subscale, in step one age and program of study accounted for $11.9 \%$ of the variance, resulting in an overall significant model, $F(4,152)=5.15, p=.001$. Adding year of study explained only $1.2 \%$ additional variance. This change was not significant. Results from the final model indicated that pharmacy students scored higher on the Cooperation subscale relative to social work students after controlling for age and year of study, $B=1.58, t=2.71, p=.008$. Year of study did not account for a significant amount of unique variance in Cooperation after controlling for age and program of study.

\section{Discussion}

Results of these analyses suggest that both field of study and year of study may be associated with perceptions of and attitudes toward interprofessional collaboration. Overall, graduate students in this sample reported positive attitudes toward collaboration. This finding is encouraging, as prior research has shown that health professionals' attitudes towards their own and other professions influence effective interprofessional collaboration (Hind et al., 2003). The results, however, revealed differences by field of study on all subscales of the IEPS. On the Prestige subscale, social work students scored lower than medicine, nursing, and pharmacy students. The Prestige subscale includes items that tap into students' perceptions of how others view their profession; for example, "People in other professions seek the advice of people in my profession." This finding raises important questions regarding the self-perceptions of social work students and the possible ways in which professional socialization may contribute to deficits in perceived value and status. This set of perceptions, which may be an artifact of the profession's historical evolution and endemic to the culture of social work, may have serious implications for both IPE and practice.

On the Cooperation subscale, social work students' scores were similar to those of nursing students, lower than pharmacy students, and slightly higher than medical students. This subscale includes items that reflect concepts likely to foster collaboration such as "Individuals in my profession are able to work closely with individuals in other professions" and "Individuals in my profession must depend on the work of people in other 
professions." On the Competency and Autonomy subscale, medical and pharmacy students rated themselves higher than social work students on items reflecting perceptions of their own competency. The finding that students from some fields of study exhibited more positive attitudes and perceptions than others is consistent with prior research (Curran et al., 2008; Hawk, Byrd, \& Killinger, 2001). These results also add to the understanding of differences among professions because most prior studies focused on or included undergraduate students as participants or lacked the necessary statistical power to detect differences between multiple health professions.

Consistent with our expectations, year of study was also associated with perceptions and attitudes towards collaboration. The findings revealed that year of graduate study was associated with variability in scores on the Prestige subscale. There was also a marginal effect of year of study on the Competency and Autonomy subscale. Specifically, first year students rated themselves higher on questions related to prestige and competency relative to more advanced students. This appears to be one of the only studies to date that examines differences in IEPS scores based on year of graduate study, as well as one of the only studies to include the dimension of prestige when considering attitudes towards interprofessional collaboration. The findings suggest that first year students have more positive perceptions of and attitudes towards interprofessionalism than more advanced students. These results are consistent with the stage-based framework of professional socialization (Miller, 2010), in which students in the anticipatory or pre-socialization stage are filled with enthusiasm and idealism towards their intended profession, which then may shift or narrow as they move further out into practice (Miller, 2013). Since administration of the IEPS occurred during student orientation and extended into the first week of graduate school classes, first year students in this study could be thought of as still in this anticipatory phase. In contrast, second year students have progressed into the formal socialization phase. In this phase, students are confronted with the realities of their profession, which may in turn result in a decrease in idealism (Miller, 2013). Participants in this study were graduate professional students, many of whom engaged in field experiences that exposed them to other professions. Thus, it is possible that this exposure to and interactions with professionals from other disciplines may have also influenced their thoughts and feelings regarding interprofessionalism.

\section{Limitations}

Results of this study should be interpreted with caution due to a number of limitations. First and foremost, response bias may be a concern due to the low response rate, as well as the possibility that students who have had interprofessional experiences may be more invested in collaboration and thus may be more likely to participate in a related research study. In addition, analysis of the sample demographic characteristics suggested that the study sample may not accurately represent the student population at large. Male students were underrepresented in the current sample, which precluded the ability to include gender as a covariate in the analyses. Future research should address this gap, particularly since some prior studies have demonstrated relationships between gender and collaboration (Van Rijnsoever \& Hessels, 2011). The fact that the sample was drawn from a single graduate school limits the generalizability of the results. Independence of observations cannot be 
guaranteed as there was no way to be certain that every student received the survey and/or that they completed the survey only once. It is also important to note that professional socialization is a complex process and this study uses only one variable as a proxy for that process. Although the results from this study demonstrate a decrease in positive perceptions of collaboration from year one to years two to four, the findings do not address potential changes in attitude over a longer period of time. It is possible that positive attitudes increase into later years of study or upon entry into a professional position.

\section{Implications}

This exploratory study begins to lay the groundwork for further research investigating the relationship between IPE and practice, and the impact of differential professional socialization. Given the global trends toward IPP, models of effective IPE are becoming increasingly important. With its history and overall ethos, social work is well-situated to not only be at the interprofessional table, but also to take a leadership role in developing and facilitating effective means of both IPE and practice.

The findings from this study raise a number of questions and highlight the need for more systematic inquiry into the role of social work in IPP and in building effective models for IPE. The results suggest that challenges associated with IPE and practice may have roots in the complex process of professional socialization. How practitioners make their way into, and then locate themselves within the context of their respective professional cultures, which are informed by long and rich histories, may be key to understanding how to maximize the benefits of IPE. Efforts to develop conceptual models that clarify pathways to interprofessional socialization will be important next steps in informing further research in this area, but also in developing innovative and effective models of IPE.

Whereas social work students in this sample perceived their profession to be of lesser prestige in the realm of IPP, medical students perceived their profession to be one that fosters less effective collaborative skills. If one considers the differences between social work and medicine historically and culturally, these findings are not at all surprising. Over the course of its lifetime in the U. S., social work has struggled with its own identity from within (Flexner, 2001), and has also struggled to be understood from without. Whereas medical doctors have garnered a reputation as experts whose decisions may have immediate life and death impact, they have also been acculturated to make those expert decisions in isolation.

Social workers also scored relatively low on their perceptions of the importance of collaboration compared to pharmacy students. This finding raises practical concerns regarding social workers' interest in and commitment to activities that require high levels of collaboration. Some of these activities, including advocacy and community organizing, are thought to be hallmarks of the social work profession. There is also the possibility that the ways in which social workers are being socialized to understand their perceived roles in interprofessional teams has bearing on the perceived importance of collaboration. Future studies should attempt to untangle the associations among how social work students and practitioners understand collaboration, their expected explicit and implicit roles in that process, and the possible intersection with perceived professional prestige. 
Further research is needed to better understand the relationship between disciplinespecific socialization and attitudes towards interprofessional collaboration over time. This study points to the potentially problematic relationships between these two constructs during the first years of graduate education; however, the nature and direction of potential changes over time is unknown. Understanding how attitudes fluctuate over time will indicate whether this pattern is resolved as a consequence of additional education and exposure or whether the circumstances require targeted intervention. Interventions may include models of IPE with explicit content about professional socialization, along with explicit and implicit curricula that simultaneously facilitate and integrate both professional and interprofessional socialization. The finding that medical, pharmacy, and nursing students evidence higher scores on prestige after controlling for year of study hints at elements of professional culture and where they fit within the larger context of professions. These fields may also be incorporating aspects of IPE in effective ways that can potentially inform efforts in social work education.

The aim of this study was to explore the relationship between aspects of professional socialization and attitudes towards interprofessional collaboration in a sample of graduate professional students. The study also explored social work students' attitudes relative to other graduate professional students with whom they are likely to interact in interprofessional settings. The results highlight both the need for efforts to build effective models of IPE that integrate professional and interprofessional socialization as well as future research in this area. With the World Health Organization's (2010) recognition that interprofessional collaboration will play a key role in "mitigating the global health workforce crisis" (p.7), it is clear that the need to grow capacity in this area is profound.

In efforts to build effective IPP capacity, social work programs must pay careful heed to how they are engaging ideas of IPE, and if or how they are working toward incorporating IPE into their curricula. These efforts should involve careful attention to the role of professional socialization and how it is manifest in the explicit curriculum, but even more elementally, in the implicit curriculum (Miller, 2013). Shedding light on the implicit factors will enhance the opportunities to build innovative mechanisms for IPE and will allow social work to maximize its characteristic capacity for building community and pragmatically and creatively bringing about change.

\section{References}

Abbott, A. A. (1988). Professional choices: Values at work. Silver Spring, MD: NASW.

American Public Health Association. (2009). Policy statement on promoting interprofessional education. Retrieved from http://www.apha.org/advocacy/policy/policysearch/default.htm?id=1374.

Bargal, D. (1981). Social values in social work: A developmental model. Journal of Sociology and Social Welfare, 8, 45-61.

Barr, H., Freeth, D., Hammick, M., Koppel, I., \& Reeves, S. (2006). The evidence base and recommendations for interprofessional education in health and social care. 
Journal of Interprofessional Care, 20(1), 75-78. doi:http://dx.doi.org/10.1080/13561820600556182

Barretti, M. (2004a). What do we know about the professional socialization of our students? Journal of Social Work Education, 40(2), 255-283. doi:http://dx.doi.org/10.1080/10437797.2004.10778493

Barretti, M. (2004b). The professional socialization of undergraduate social work students. Journal of Baccalaureate Social Work, 9(2), 9-30.

Boden, D., Borrego, M., \& Newswander, L. K. (2011). Student socialization in interdisciplinary doctoral education. Higher Education, 62, 741-755. doi:http://dx.doi.org/10.1007/s10734-011-9415-1

Bronstein, L. R., Mizrahi, T., Korazim - Korosy, Y., \& McPhee, D. (2010). Interdisciplinary collaboration in social work education in the U.S.A., Israel, and Canada: Deans' and directors' perspectives. International Social Work, 53(4), 457473. doi:http://dx.doi.org/10.1177/0020872809358399

Buring, S. M., Bhushan, A., Broeseker, A., Conway, S., Duncan-Hewitt, W., Hansen, L., \& Westberg, S. (2009). Interprofessional education: Definitions, student competencies, and guidelines for implementation. American Journal of Pharmaceutical Education, 73(4) 1-11. doi:http://dx.doi.org/10.5688/aj730460

Clark, P. G. (1997). Values in health care professional socialization: Implications for geriatric education in interdisciplinary teamwork. The Gerontologist, 37(4), 441-451. doi:http://dx.doi.org/10.1093/geront/37.4.441

Clark, S. M., \& Corcoran, M. (1986). Perspectives on the professional socialization of women faculty: A case of accumulative disadvantage? The Journal of Higher Education, 57, 20-43. doi:http://dx.doi.org/10.2307/1981464

Council on Social Work Education [CSWE]. (2012). Setting priorities, serving the nation: A shared agenda for social work education. Retrieved from http://www.cswe.org/File.aspx?id=63558

CSWE. (2015). Educational policy and accreditation standards. Retrieved from http://www.cswe.org/Accreditation/EPASRevision.aspx

Couturier, Y., Gagnon, D., Carrier, S., \& Etheridge, F. (2008). The interdisciplinary condition of work in relational professions of the health and social care field: A theoretical standpoint. Journal of Interprofessional Care, 22(4), 341351.doi:http://dx.doi.org/10.1080/13561820802190616

Curran, V. R., Sharpe, D., Forristall, J., \& Flynn, K. (2008). Attitudes of health sciences students towards interprofessional teamwork and education. Learning in Health and Social Care, 7(3), 146-156. doi:http://dx.doi.org/10.1111/j.1473-6861.2008.00184.x

Flexner, A. (2001). Is social work a profession? Research on Social Work Practice, 11(2), 152-165. [Originally published 1915] doi:http://dx.doi.org/10.1177/104973150101100202 
Freshman, B., Rubino, L., \& Chassiakos, Y. (2010). Collaboration across the disciplines in health care. Sudbury, MA: Jones \& Bartlet.

Hall, P. (2005). Interprofessional teamwork: Professional cultures as barriers. Journal of Interprofessional Care, 19(sup1), 188-196. doi:http://dx.doi.org/10.1080/13561820500081745

Hawk, C., Buckwalter, K., Byrd, L., Cigelman, S., Dorfman, L., \& Ferguson, K. (2002). Health professions students' perceptions of interprofessional relationships. Academic Medicine, 77(4), 354-357. doi:http://dx.doi.org/10.1097/00001888-200204000-00020

Hawk, C., Byrd, L., \& Killinger, L. Z. (2001). Evaluation of a geriatrics course emphasizing interdisciplinary issues for chiropractic students. Journal of Gerontological Nursing, 27(7), 6-12. doi:http://dx.doi.org/10.3928/0098-9134$\underline{\text { 20010701-07 }}$

Health Resources Services Administration [HRSA]. (2012). New coordinating center will promote interprofessional education and collaborative practice in health care [Press release]. Retrieved from http://www.hrsa.gov/about/news/pressreleases/120914interprofessional.html

Hind, M., Norman, I., Cooper, S., Gill, E., Hilton, R., Judd, P., \& Jones, S. (2003). Interprofessional perceptions of health care students. Journal of Interprofessional Care, 17(1), 21-34. doi:http://dx.doi.org/10.1080/1356182021000044120

Institute of Medicine. (2003). Health professions education: A bridge to quality. Washington, DC: National Academy Press.

Kenny, M., Sparks, E., \& Jackson, J. (2007). Social justice through interprofessional university-school collaboration. In E. Aldarondo (Ed.), Promoting social justice through mental health practice (pp. 313-335). Mahwah, NJ: Lawrence Erlbaum Associates.

Ko, J., Bailey-Kloch, M., \& Kim, K. (2014). Interprofessional experiences and attitudes toward interprofessional health care teams among health sciences students. Social Work in Health Care, 53(6), 552-567. doi:http://dx.doi.org/10.1080/00981389.2014.903884

Leipzig, R. M., Hyer, K., Ek, K., Wallenstein, S., Vezina, M. L., Fairchild, S., ...\& Howe, J. L. (2002). Attitudes toward working on interdisciplinary healthcare teams: A comparison by discipline. Journal of the American Geriatrics Society, 50(6), 11411148. doi:http://dx.doi.org/10.1046/j.1532-5415.2002.50274.x

Leitch, J. (2014). Exploring psychometric properties of the Interdisciplinary Education Perception Scale in health graduate students. Journal of Interprofessional Care, 28(1), 52-57. doi:http://dx.doi.org/10.3109/13561820.2013.820691

Luecht, R. M., Madsen, M. K., Taugher, M. P., \& Petterson, B. J. (1990). Assessing professional perceptions: Design and validation of an Interdisciplinary Education Perception Scale. Journal of Allied Health, 19, 181-191. 
Mabry, P. L., Olster, D. H., Morgan, G. D., \& Abrams, D. B. (2008). Interdisciplinarity and systems science to improve population health: A view from the NIH Office of Behavioral and Social Sciences Research. American Journal of Preventive Medicine, 35(2 Suppl), S211-224. doi:http://dx.doi.org/10.1016/j.amepre.2008.05.018

Matto, H. C., \& Strolin-Goltzman, J. (2010). Integrating social neuroscience and social work: Innovations for advancing practice-based research. Social Work, 55(2), 147156. doi:http://dx.doi.org/10.1093/sw/55.2.147

McFadyen, A. K., Maclaren, W. M., \& Webster, V. S. (2007). The Interdisciplinary Education Perception Scale (IEPS): An alternative remodeled subscale structure and its reliability. Journal of Interprofessional Care, 21(4), 433-443. doi:http://dx.doi.org/10.1080/13561820701352531

McPherson, K., Headrick, L., \& Moss, F. (2001). Working and learning together: Good quality care depends on it, but how can we achieve it? Quality \& Safety in Health Care, 10(Suppl II), ii46-ii53. doi:http://dx.doi.org/10.1136/qhc.0100046

Miller, S. E. (2010). A conceptual framework for the professional socialization of social workers. Journal of Human Behavior in the Social Environment, 20, 924-938. doi:http://dx.doi.org/10.1080/10911351003751934

Miller, S. E. (2013). Professional socialization: A bridge between the explicit and implicit curricula. Journal of Social Work Education, 49, 368-386.

Pecukonis, E., Doyle, O., Acquavita, S., Aparicio, E., Gibbons, M., \& Vanidestine, T. (2013). Interprofessional leadership training in MCH social work. Social Work in Health Care, 52, 625-641. doi:http://dx.doi.org/10.1080/00981389.2013.792913

Reeves, S., Zwarenstein, M., Goldman, J., Barr, H., Freeth, D., Koppel, I., \& Hammick, M. (2010). The effectiveness of interprofessional education: Key findings from a new systematic review. Journal of Interprofessional Care, 24(3), 230-241. doi:http://dx.doi.org/10.3109/13561820903163405

Sharland, E. (2012). All together now? Building disciplinary and inter-disciplinary research capacity in social work and social care. British Journal of Social Work, 42, 208-226. doi:http://dx.doi.org/10.1093/bjsw/bcr061

Shuval, J. T. (1980). Entering medicine: The dynamics of transition. A seven year study of medical education in Israel. Oxford, England: Pergamon.

Simms, D. (2011). Reconstructing professional identity for professional and interprofessional practice: A mixed methods study of joint training programmes in learning disability nursing and social work. Journal of Interprofessional Care, 25, 265-271. doi:http://dx.doi.org/10.3109/13561820.2011.571352

Syme, S. L. (2008). The science of team science: Assessing the value of transdisciplinary research. American Journal of Preventive Medicine, 35, 94-95. doi:http://dx.doi.org/10.1016/j.amepre.2008.05.017 
U. S. Bureau of Labor Statistics. (2014). Labor force statistics from the current population survey. Retrieved from http://www.bls.gov/cps/cpsaat11.htm

Van Rijnsoever, F. J., \& Hessels, L. K. (2011). Factors associated with disciplinary and interdisciplinary research collaboration. Research Policy, 40, 463-472. doi:http://dx.doi.org/10.1016/j.respol.2010.11.001

Vaughan, B., Macfarlane, C., Dentry, T., \& Mendoza, G. (2014). The Interdisciplinary Education Perception Scale (IEPS): Which factor structure? Education in Medicine Journal, 6(3), e67-e71. doi:http://dx.doi.org/10.5959/eimj.v6i3.259

Williams, B., \& Webb, V. (2013). Examining the measurement properties of the Interdisciplinary Education Perception Scale (IEPS) in paramedic education. Nurse Education Today, 33(9), 981-985. doi:http://dx.doi.org/10.1016/j.nedt.2012.10.015

World Health Organization [WHO]. (2010). Framework for action on interprofessional education \& collaborative practice. Retrieved from http://whqlibdoc.who.int/hq/2010/WHO_HRH_HPN_10.3_eng.pdf

\section{Author note:}

Address correspondence to: Allison West, Department of Population, Family, and Reproductive Health, Johns Hopkins Bloomberg School of Public Health, 615 N. Wolfe Street, Baltimore, MD 21205, awest25@jhu.edu. 